Galapagar con el colateral de Villa del Prado, realizado poco antes y quizá de tamaño similar, por el que se pagaron a Juan de Borgoña 34.027 maravedises ${ }^{17}$.

LuIS ZOLLE BETEGóN

Universidad Autónoma de Madrid

\title{
ARQUETA FLAMENCA DE LOS CINCO SENTIDOS EN ALCALÁ DE HENARES
}

En la Iglesia Magistral de Alcalá de Henares se conserva un precioso cofrecillo de marfil, una de las piezas más finas de las conservadas en España de por los años finales del siglo XVI. De base rectangular, presenta la tapa combada y aparece recubierta de planchas de marfil sobre armazón metálico. En cada frente y en la tapa se representa una escena sobre un precioso fondo de decoración manierista enmarcada por simples molduras. La estructura metálica, refuerza al exterior, con molduras rectilíneas, las superficies talladas en el marfil de los frentes, laterales y tapa. Asienta sobre bolas, con bella cerradura y asas a los lados.

En un principio los temas representados en sus superficies plantearon problemas de identificación por lo que se analizaron con detenimiento. La plaqueta del frente de la arqueta representa una bella figura femenina sentada delante de una gruesa columna mirándose en un espejo. En el primer plano un águila con las alas desplegadas y de tamaño desproporcionado en relación al de la figura vuelve su cabeza hacia ella. Un fondo de paisaje marítimo, con una barquichuela con velas y a su derecha arquitecturas simulando una ciudad, completan la escena. En la del reverso se representa una figura sentada con el brazo extendido cuya mano picotea un pájaro. Al fondo, a la derecha, árboles cuyas ramas cobijan una telaraña. Una tortuga en primer término y a la izquierda una barca encallada en la orilla con las redes en la borda, sobre el agua que al fondo delinea la costa y una isla con edificios humeantes. En la tapa una figura femenina coronada de una guirnalda de flores acerca hacia sí unas ramas de un jarrón sosteniendo otro semejante con flores sobre sus piernas. a su derecha dos perros, uno de los cuales apoya sus patas en el recipiente olisqueando las flores, destacando sobre un fondo de árboles y edificios. En una de las plaquetas de los laterales, una fina figura de mujer toca el laúd observada por un ciervo. A sus pies una trompeta doblada en $« \mathrm{~S} » \mathrm{y}$ una viola de «braccio» con una partitura musical abierta ${ }^{1}$. La escena del otro lateral representa una mujer con los senos desnudos, sentada y comiendo, mientras sostiene con su mano un cesto de frutos ante el que se representa una jarra y una copa. En el ángulo inferior derecho un mono, también comiendo, aparece ante otra cesta con alimentos (Figs. 15-16, 22-23).

La identificación de estas escenas con la representación de los Cinco Sentidos ha sido relativamente fácil por los símbolos que las acompañan. En la primera descrita, el espejo en el que se mira la figura y el águila, ave cuya vista aventaja a las de su especie y que puede mirar al sol de frente, aluden al sentido de la Vista que puede deleitarse en la contemplación de un amplio paisaje. La representación del Tacto por el pájaro que pica la mano y produce dolor fue en realidad una innovación iconográfica renacentista que identifica en esta sensación la más exacta re-

\footnotetext{
17 Ibidem.

${ }^{1}$ La doctora Rosario Álvarez, Catedrático de Musicología de la Universidad de la Laguna, me indicó amablemente el tipo de instrumentos representados.
} 


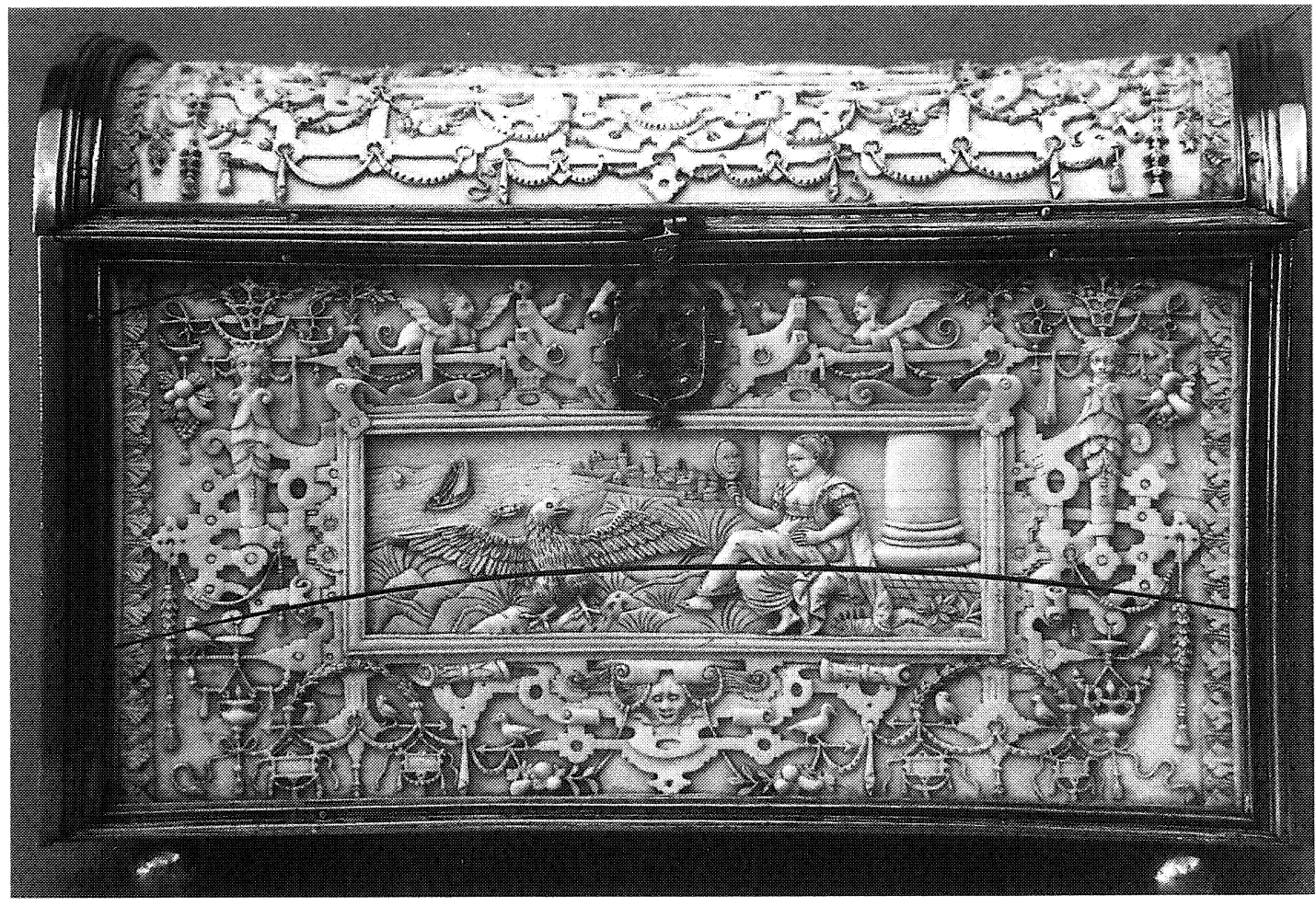

15

16
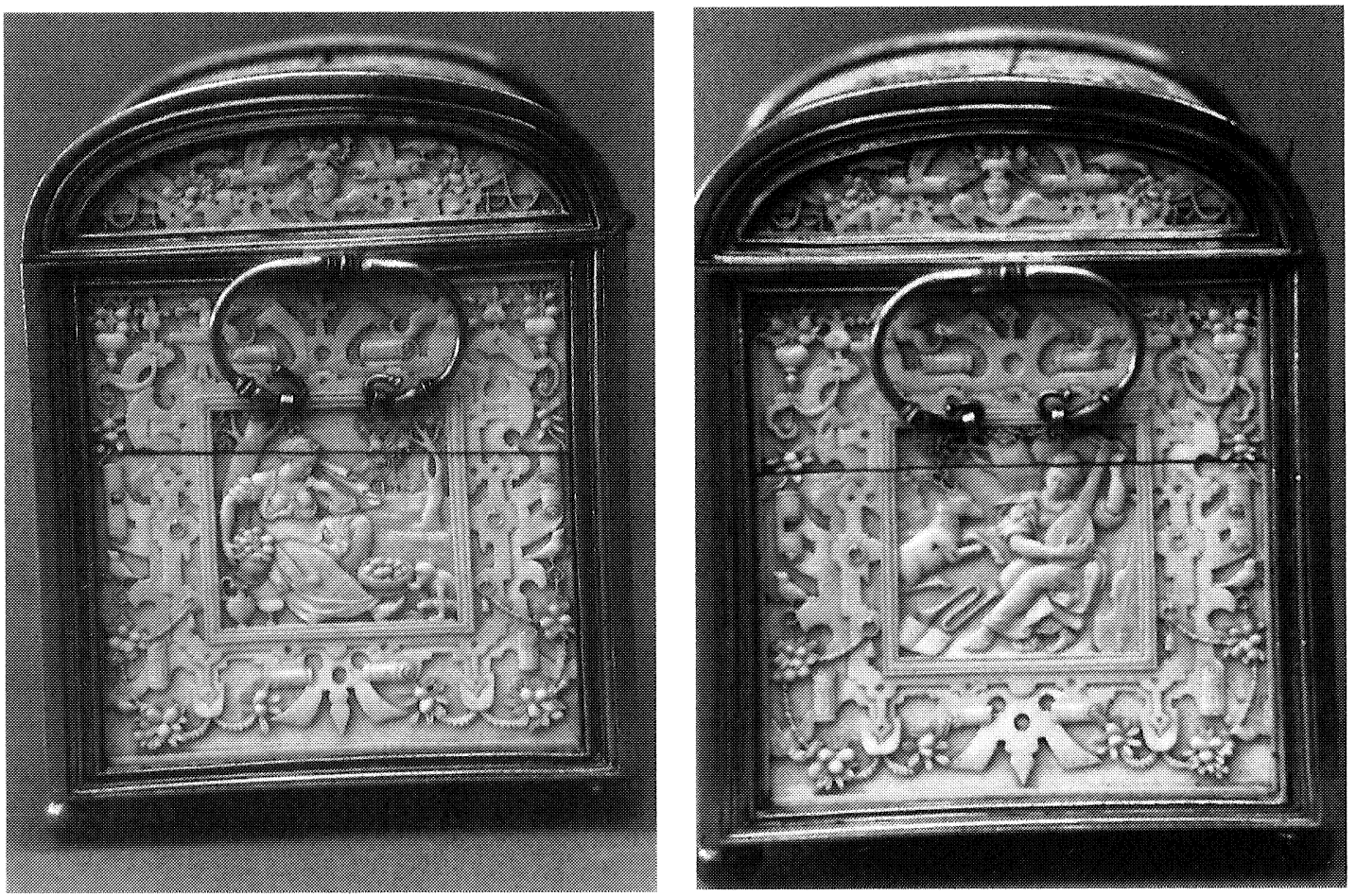

Fig. 15. La Vista. Fig. 16. El Gusto, Fig. 17. El Oído, de la arqueta de marfil. Alcalá de Henares (Madrid). Iglesia Magistral. 
presentación de este sentido, en cierto modo en su aspecto sexual. La tortuga se asocia a este sentido como símbolo del amor doméstico y por su sensibilidad al tacto que la hace esconder su cabeza bajo el caparazón al menor estímulo. La araña que también se retrae al menor contacto simboliza, además, la fábula de aracne, la mítica tejedora. Los instrumentos musicales que acompañan a la figura femenina tocando el laúd y la repesentación del Ciervo, animal de finísimo Oído, simbolizan este sentido. El Olfato se identifica por el olor de las flores y los animales que como el perro poseen agudizado este sentido. Tampoco presenta complicaciones la identificación del Gusto por la figura representada comiendo junto al mono que se caracteriza entre otros aspectos por su glotonería.

La evolución iconográfica de estos temas, estudiada recientemente por Konêcny desde su definición conceptual por Aristóteles y su representación a través del tiempo, tuvo su culminación en la interpretación de Frans Floris de 1561, cuyos dibujos, como el del Tacto del Museo de Budapest, fueron grabados por Cort. Impresos por Cok tuvieron una amplia difusión como muestran las escenas de esta arqueta directamente inspiradas en ellos ${ }^{2}$ (Figs. 18-20, 24-25).

Dentro de la específica talla del marfil, de su condicionamiento material y del artista que la realizó, la escena de la Vista se ajusta exactamente a su modelo aunque su formato exige un alargamiento de la composición que se consigue volviendo la figura en una posición de perfil, aunque en su misma actitud, con un brazo apoyado en la cintura y el otro sosteniendo en la mano el espejo. El águila cambia su posición de tres cuartos por la de frente, con las alas desplegadas que rellenan el espacio vacío. Se ha eliminado el sol en lo alto. La escena del Tacto aparece invertida respecto a su modelo grabado y por lo mismo, en la misma posición que aparece en el citado dibujo de Budapest. Se utiliza el mismo recurso de colocar la figura, que en la arqueta pudiera ser masculina, de perfil pero con la misma disposición de los brazos y los mismos atributos de la telaraña en el árbol que no aparece en el dibujo original, la barca con las redes, elemento del que no se conoce su significado y que quizás aluda al agua del mar como sustitución de la lluvia que aparece en otras interpretaciones de este sentido. La tortuga en primer término, de significado conocido, y el edificio humeante al fondo, tampoco visible en el dibujo de Floris y sin explicación en los textos consultados. El Olfato de la arqueta presenta composición algo distinta a la de Frans Floris pero integra los mismos atributos, la mujer oliendo las flores de un jarrón. La figura del perrillo que pone sus patas sobre el otro jarrón sostenido por la mujer sobre su pierna desnuda, está tomado del grabado de Cort de la composición de Floris pero en ésta la figura femenina inclina su cabeza. La de la arqueta presenta posición más similar a la que aparece en la interpretación del tema por Martín de Vos, de su serie de los Cinco Sentidos asociada a temas evangélicos y grabada por Collaert, como por ejemplo en el detalle de la túnica recogida sobre la pierna ${ }^{3}$. La representación del Oído se ajusta literalmente a la composición de Floris pues su formato cuadrado facilita la copia directa. La figura del ciervo, con gran cornamenta, aparece invertida y en el marfil cruza sus patas delanteras siendo distintos y más numerosos los instrumentos musicales representados en el grabado de la composición del pintor flamenco. La escena del Gusto de Floris presenta una figura femenina en posición similar a la representada en el marfil en el que la cesta de frutos aparece en el suelo a la izquierda y el mono, también a la altura de sus pies, a la derecha. Es muy curioso que en la arqueta se represente un granado que no aparece en la composición del pintor flamenco, pues pudiera aludir al Emblema de Soto (1599) del «Deseo humano» que se in-

\footnotetext{
${ }^{2}$ Konëcny Arthur, Los Cinco Sentidos y el Arte. Madrid, Museo del Prado, 1997. Cat. pp. 108-111. Fiamminghi a Roma, 1508-1608. Artistes des Pays Bas et de la Principauté de Liège à Rome à la Renaissance. Bruxelles, Palais des Beaux Arts, 24 février-21 mai 1995; Rome, Palazzo delle Esposizioni, 7 junio-4 septembre, 1995. Commissaires Nicole Dacos, Bert N. Meijer, p. 91 Frans Floris, Alegoria del Tacto.

${ }^{3}$ Konëcny, cit., pp. 112-1115.
} 


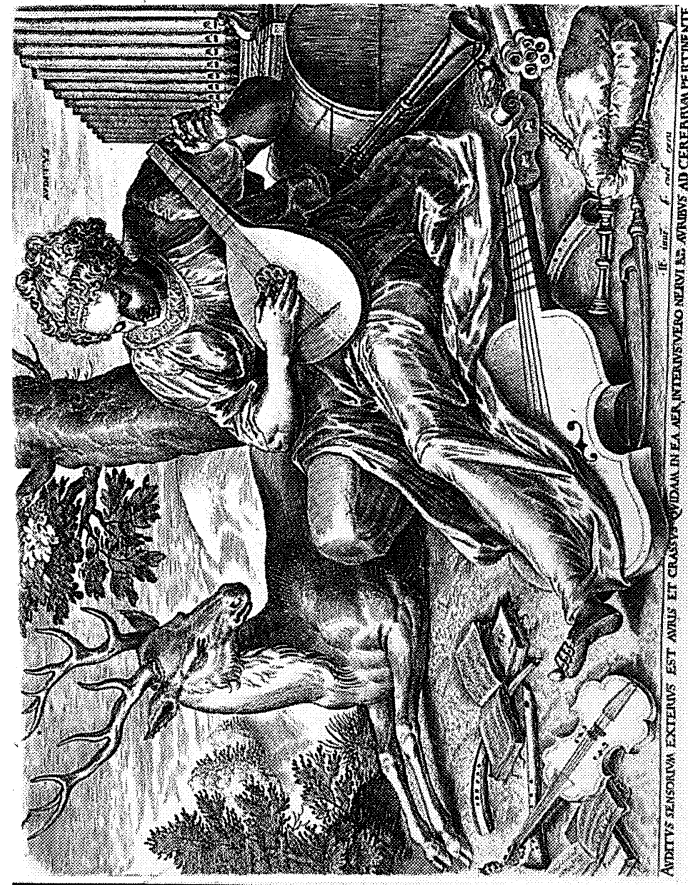

2

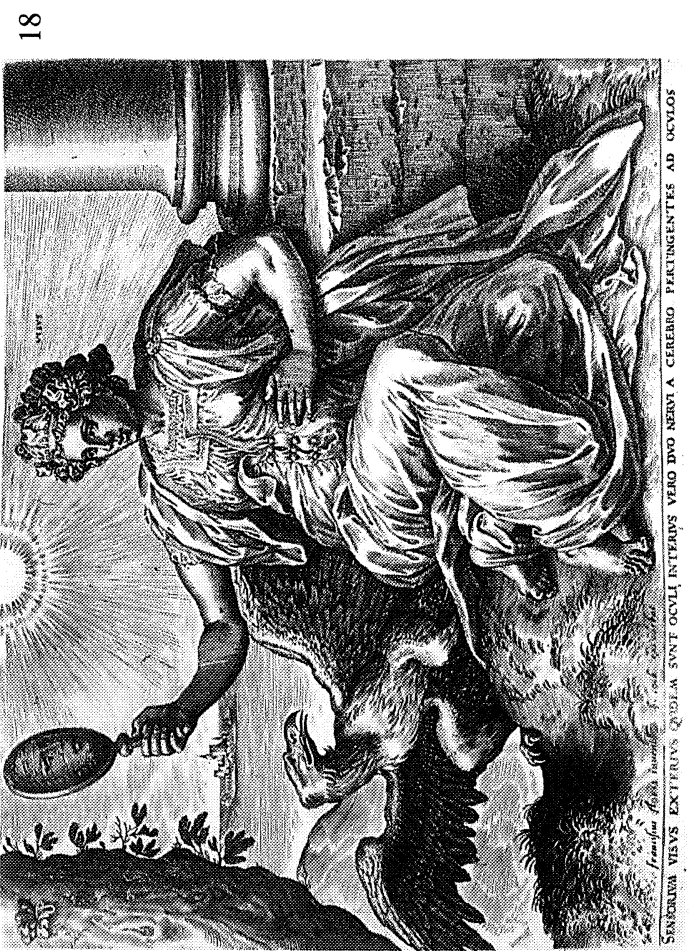

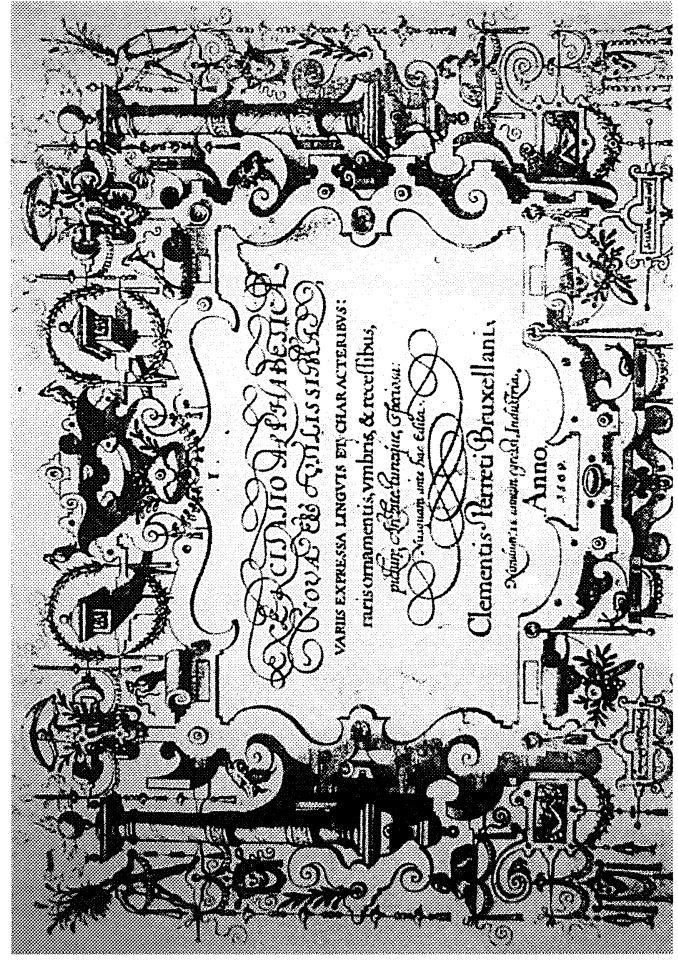

$\bar{\sim}$

กิ

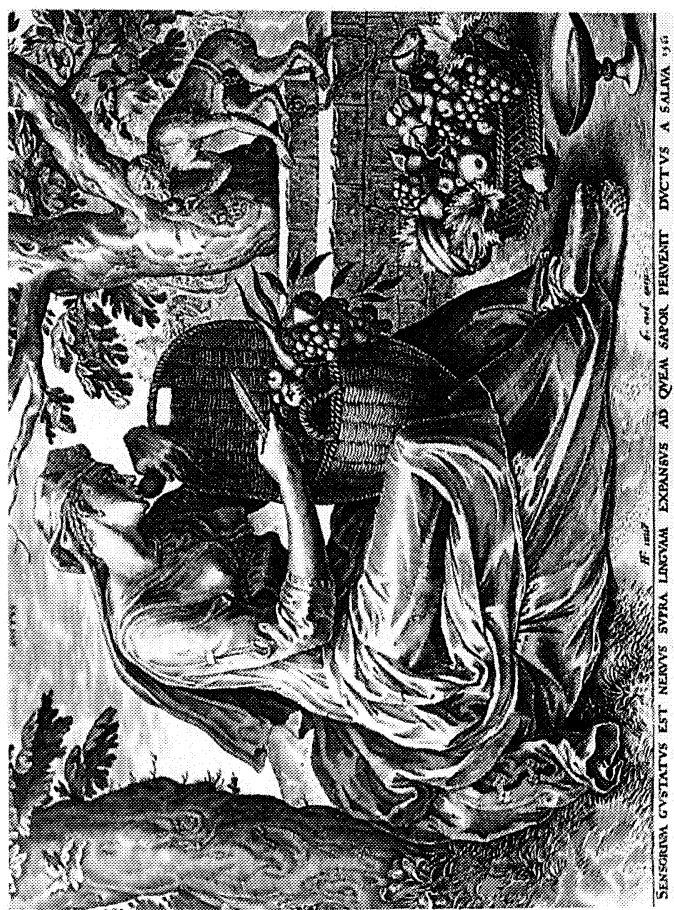

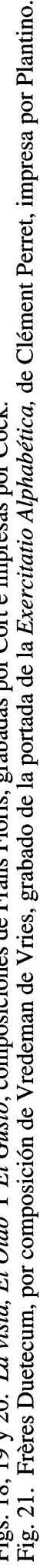


terpreta por un mono que queriendo alcanzar la fruta de este árbol, la desecha al probarlo, por su amargo sabor ${ }^{4}$.

Respecto a la magnífica decoración que recubre toda la arqueta alrededor de estas escenas, se han localizado fuentes próximas que apuntan al mismo arte flamenco del círculo de Frans Floris y más especialmente a la obra de su hermano Cornelis Floris. Éste también famoso artista flamenco, a su vuelta de Roma en los años de hacia 1550-1557, edita una serie de motivos ornamentales en los que, como explica Osten ${ }^{5}$, desarrolla «un fantástico microcosmos de vasos, cartelas, máscaras y motivos derivados de la escuela de Fontainebleau, imitando el agudo corte del metal». Fue, después de Coecke y al tiempo que Cornelis Bos, el introductor del gusto por un específico grutesco que marca con su impronta todo el arte flamenco posterior ${ }^{6}$.

De hecho, comparados en su conjunto y detalle a detalle los motivos de la arqueta con los repertorios grabados flamencos de este círculo, se ha advertido una clara relación de los representados en marfil con las series que de un modo más o menos diecto entroncan con el arte de Cornelis Floris, como puede advertirse claramente en el bellísimo y original conjunto ornamental de Vredeman de Vries, pintor y arquitecto de origen alemán que desarrolla gran parte de su actividad artística precisamente en Amberes donde ejercía su maestrazgo Cornelis Floris, y prácticamente por sus mismos años ${ }^{7}$.

Delen reprodujo unos grabados de los Hermanos Duetecum, tomados de Vries, que presentan muchos de los motivos de la arqueta, destacando el repertorio de ruedecillas dentadas que como mecanismos de relojería aparecen a un lado y otro de la escena de la Vista ${ }^{8}$. En el grabado de los Duetecum, portada del libro de la «Exercitatio Alphabética», publicada por Plantinus en 1569 (Fig. 7), también aparece otros motivos como las guirnaldas de fino dibujo, pajarillos de delicadas siluetas destacando sobre un fondo difuso y abigarrado de placas con roleos de pronunciadas curvas, motivo cuya llegada a España se ha detectado precisamente por estos años medios del siglo XVI destacando su procedencia de la escuela de Fontainebleau y cuyos afilados bordes recuerdan lo que se ha dicho del arte de Cornelis Floris cuyos grabados aparentan un corte metálico ${ }^{9}$. En el extemo inferior del Olfato, a un lado y otro de la barra con guirnaldas, aparecen dos bucráneos que en el grabado de los Duetecum se representan en la curva de las volutas de la parte superior del encuadramiento, y en su parte inferior presenta esfinges que, con alas, aparecen sobre la escena de la Vista, a un lado y otro de la cerradura. Las cariátides a sus lados o los finos querubes de la escena del Tacto son motivos típicos de la escuela de Fontainebleau como se ve en grabados de Fantuzzi, Mignon, etc., pero también en los del flamenco Cornelis Bos, mucho más carnosos y en composiciones distintas a las decorativas de la arqueta. Los jarrones panzzudos de Cornelis Floris aparecen representados en las historias de marfil pero son

\footnotetext{
${ }^{4}$ Henkel Arthur und Schöne, Albrecht. Emblemata, Handbuch zur Sinnbildkunst des XVI und XVII Jahrhunderts. Herausgegeben..Stuttgart, 19667, p. 435 (en Affe -mono). Soto. Emblemas moralizados, 1599. Aunque la fecha de edición sea quizás posterior a la realización de la arqueta es problable que en estos años de saber emblemático se conociera el precedente de este concreto emblema.

${ }^{5}$ Roggen D. and Withof, J., «Cornelis Floris», en Gentsche Bijdraghen tot de Kunstgeschiedenis, VIII, 1942, pp. 79171. Osten, G. van, Painting and sculpture in Germany and Netherlands (1500-1600). By..and H. Vey London, Pelican History of Art, 1969. Huysmans, Antoinette, «Die Grabmonumenten von Cornelis Floris», en Revue belge d'archéologie et d'histoire de l'art, 1987, pp. 01-1222.

${ }^{6}$ Sune Schele, Cornelis Bos. A study of the origins of the Netherland grotesque. Stockholm, 1655.

7 Vid. nota 4 y Berliner, Rudolf, Modelos ornamentales de los siglos XV al XVIIII. I texto, II láminas. Barcelona, Labor (1928), por ejemplo, láms. 160-163 (Cornelis Floris): láms. 166-167.

${ }^{8}$ Delen, A. J. J., Hisstoire de la gravure dans les Pays Bas et dans les provinces belges des origines jusqu'au Xvillè siècle. I. Des origines à 1500. II. Le Xviè siècle. Les graveurs d'estampes. Les graveeurs illustrateeurs. Parrrrrís, 1924-35, 3 vols. II. Les graveurs d'estampes.

${ }^{9}$ Alcolea, Santiago, «Penetración y desarrollo de un repertorio ornamental en nuestro siglo XVI: los temas de placas con roleos», en Academia, 1. ${ }^{\text {er }}$ semestre, 1993, n. ${ }^{0} 76$, pp. 204-241.
} 

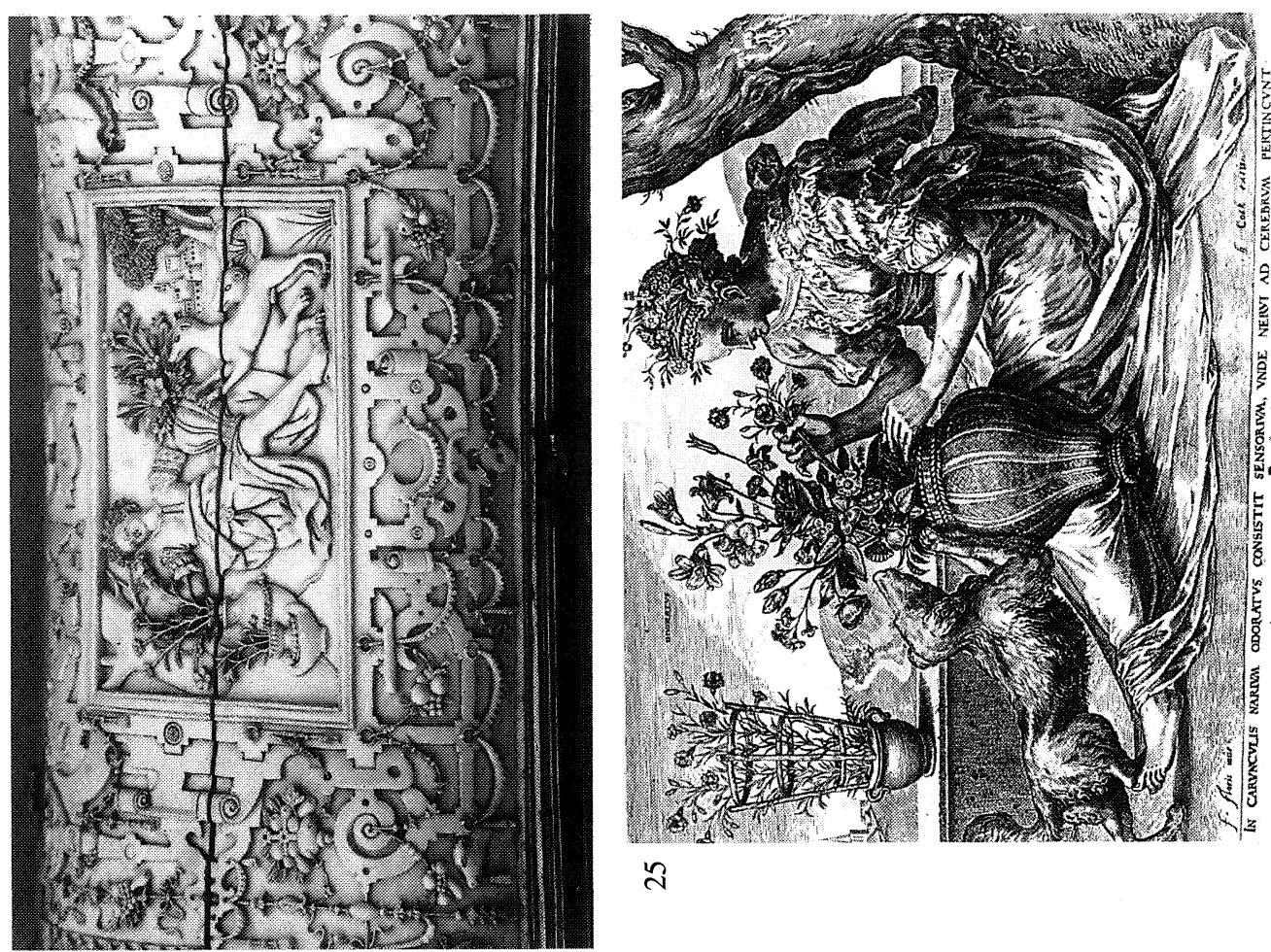

\section{$\sqrt[2]{ }$}

ה

N

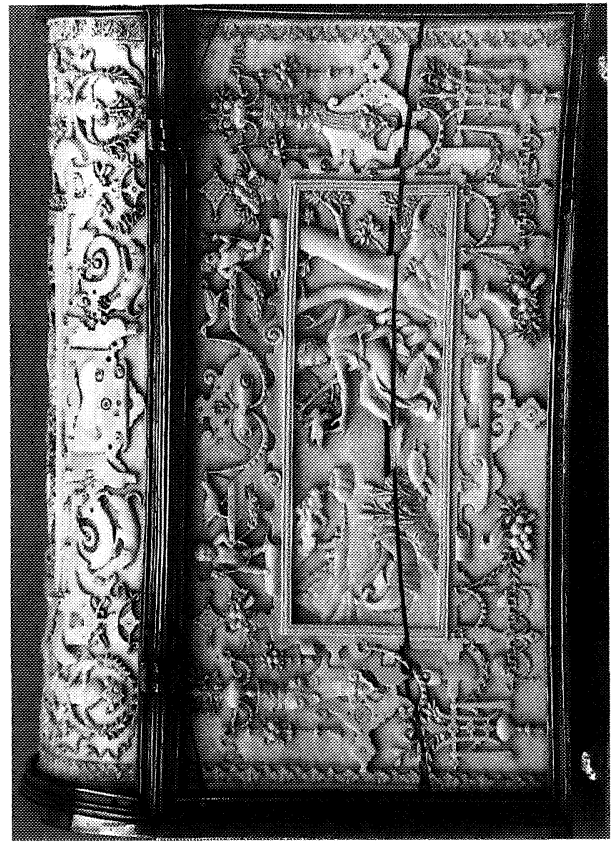

ป

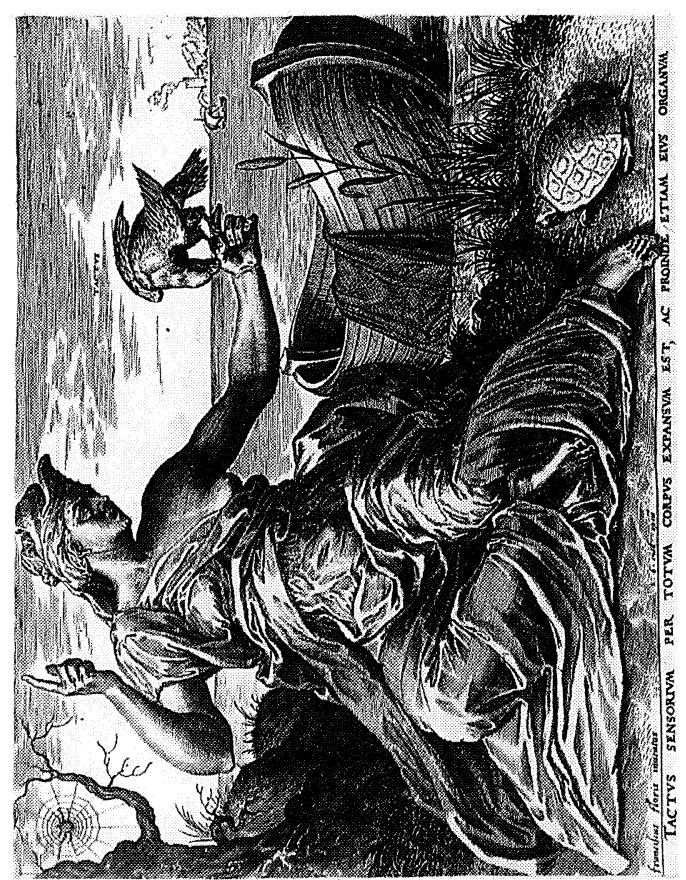

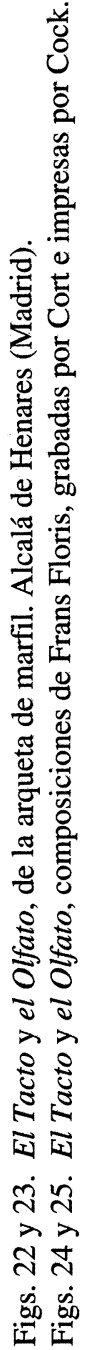


los grabados de la obra de Vredeman de Vries o de sus intérpetes la fuente más próxima de toda la decoración de la arqueta, incluida su extraña cariátide ${ }^{10}$.

El estudio estilístico de esta arqueta también confirma su adscripción a un taller flamenco italianizado de la segunda mitad del siglo XVI. Los autores que se ocuparon de ella fluctuaron entre su clasificación como obra francesa o como obra italiana, lo cual no cntradice la realidad pues su arte denuncia la influencia italiana a través de sus modelos interpretados en la escuela de Fontinebleau y de sus fuentes gráficas más próximas, tanto a los grabados de la obra de Frans Floris como a los mencionados de su hermano Cornelis que en su estancia en Italia había asimilado los principios del manierismo italiano ${ }^{11}$.

Pero también su talla apunta esta influencia que domina la eboraria flamenca de estos años y en concreto, a falta de otros nombres de actividad conocida en este campo, puede relacionarse con el arte de Jacob Cornelisz Cobaert, más conocido por el nombre de Coppe, flamenco que trabajaba en Roma en el taller de Gugliemo della Porta y que se destacó como artífice de pequeñas figuras en cera para trabajos de orfebrería y al que se le atribuye la realizaciónde varios relieves de marfil, ovales, representando temas de la Metamorfosis de Ovidio. Estos relieves, muy planos, de finas figurillas femeninas algo cortas de proporciones y que ocupan todo el espacio de la escena con un fondo amplio de paisaje, recuerdan las que aparecen en las historias talladas en la arqueta de Alcalá de Henares, sin que su semejanza sea tan clara como para poderle atribuir la obra ${ }^{12}$. En realidad su obra documentada y localizada en materiales metaálicos difiere de los relieves de marfil que se le atribuyen pero en cualquier caso, su actividad en la pequeña escultura sugiere su probable conocimiento del arte de la eboraria en unos años que los artistas nórdicos en su viaje obligado a Italia se dedican a su práctica en casos como medio de vida hasta abrirse camino en otros campos, que como ha aclarado Gramberg consiguió con su monumental grupo de San Mateo y el Ángel, terminado por Ferrucci ${ }^{13}$. Es lógico que fuera Coppe u otro de los numerosos artistas flamencos trabajando en Italia los que copiaran antes la obra de Frans Floris, difundida rápidamente por el grabado y muy a propósito para su representación en pequeños objetos con relieves y que, asimismo, conocieran la serie decorativa de Cornelis o incluso de Vredeman de Vries que divulgaban los grabados.

Como conclusión puede decirse que esta fina arqueta de la Sagrada Espina que representa los Cinco Sentidos es de arte flamenco de la segunda mitad del siglo XVI. Su artífice conoció los grabados de los Cinco Sentidos de Frans Floris y los adaptó al formato de las plaquetas de marfil inspirándose para su decoración en los grabados de Cornelis Floris y más posiblemente en los de la obra de Vredeman de Vries. La técnica en la que están trabajados los relieves recuerda el arte de la obra en marfil atribuida al flamenco Coppe, quizás sin demasiado fundamento esti-

\footnotetext{
${ }^{10}$ Sune, cit. y Berliner, cit. lám. 167, que deriva de la de Bos, lám. 153.

" Puyvelde, Leo van, La peinture flamande au siècle de Bosch et Brueghel. Bruxelles, Elsevier, 1962: Frans Floris, pp. 332-341. Van de Velde, Frans Floris (1519/1520-1570). Leven en werken. Brussel, Paleis der Academiën, 1975, 2 vols. Sobre Cornelis Floris, vid. nota 5 y Berliner, cit.

${ }^{12}$ Sobre Coppe: Baglione, Gio Romano, Le Vite de Pittori, scultori et architetti. Dal Pontificato di Gregorio XI del 1572. In fino a tempi di Papa Urbano Ottavo nel 1642. Facsimile dell'edizione del 1642, Roma, 1935, p. 100. Bever. Geneviève, Les «Taylleurs d'Yvoire» de la Renaissance au XIX siècle. Bruxelles, 1946, p. 56, I; Fiamminghi a Roma, cit.: Jacob Conelisz Cobaert di Cope, Fiamingo, p. 144; se le atribuye un grupo de la Piedad muy diferente a las escenas de la Metamorfosis que estudia la Bever (vid. supra).

${ }_{13}$ Porta, Guglielmo della, Düsseldorfer Skizzenbücher des... Edición por Werner Gramberg. Berlín, 1964 (reedición, 1998). Theuerkauff, Christian, Elfenbein. Sammlung Reiner Winkler, I, München, 1984, II. München, 1994: I, cat. 61 y II cat. 24: da noticias de las plaquetas en bronce, por dibujos de Guglielmo de la Porta, conservadas en el Museo de Bellas Artes de Viena, obras de Cope que inspiraron los relieves de marfil estudiados. Gramberg, Werner, «Guglielmo della Porta, Coppe Fiammingo und Antonio Gentile da Faenza», en Jahrbuch der Hamburger Kunstsammlungen, V, 1960, recogido por Pressouyre, Sylvie, Nicolas Cordier. Recherches sur la sculpture à Rome autour du 1600. Ecole Française de Rome, 1984, que estudia y reproduce, pp. 211-213, lám. 273, la escultura de San Mateo y el Ángel.
} 
lístico aunque con una cierta base documental. No se conoce su procedencia exacta pues aunque se sabe que fue regalada por el Cardenal García de Loaisa, la biografía del donante no aclara el posible lugar de donde vino que por lo dicho tanto podía ser Italia como Flandes.

Según los Anales Complutense, manuscrito que abarca hasta el año de 1627, en 1599 el Cardenal García de Loaisa había regalado una de las dos Sagradas Espinas que se conservaban en la Magistral, traída de Roma. Se conservaba en un cofrecito de preciosas labores y cuyo precio podía calcularse en unos 2.000 ducados ${ }^{14}$ pero en el Inventario de los bienes de sus casas en Madrid ${ }^{15}$ no aparece noticia alguna referente a la reliquia ni tampoco datos que permitan identificar la arquilla. Puede pensarse con toda probabilidad que la reliquia y la arquilla se conservaban en Alcalá de donde, según una nota al margen del Inventario ${ }^{16}$, se esperaba la relación de los bienes que el Cardenal tenía en aquella ciudad para completar la que se había hecho en Madrid.

En efecto, la donación de una de las dos Sagradas Espinas que guarda en su interior por el Cardenal García de Loaisa a finales del siglo XVI sugiere la adquisición por el mismo de la arqueta en estos mismos años. Como los temas que la decoran no son propios de un relicario, puede pensarse que no fue obra de encargo sino adquirido para la ocasión. Las historias de los Cinco Sentidos representadas pudieran precisar un primitivo uso no identificado, pues aunque en casos se conocen enseres similares regalados a novias famosas, como el de la Catedral de Graz que en torno a 1477 enriqueció el ajuar de Paola Gonzaga en sus esponsales con el Conde Leonardo Gorizia, su decoración no es la misma pues fue adornada de los «Triunfos» de Petrarca, derivados de prototipos mantegnescos ${ }^{17}$.

El año de 1893 vuelve a recordarse la arqueta de marfil como «un precioso cofrecito de marfil con dos Sagradas Espinas, una de las cuales era donación del Cardenal García de Loaisa de los tiempos de Felipe II». Tormo, poco después, aludió a su arte sugiriendo su posible origen francés, juicio que recoge el «Inventario artístico de la provincia de Madrid». El año de 1926 se exhibió en la Exposición de Arte religioso de Alcalá» como obra del siglo XVI del Renacimiento italiano, clasificación que no recogimos en su mera cita en 1984. La Exposición de «Madrid en el Renacimiento» de 1986 la presentó consignando únicamente su estructura de placas de marfil sobre armadura metálica ${ }^{18}$.

MARGARITA M. ESTELLA Departamento de Historia del Arte del CSIC

\footnotetext{
14 Anales Complutenses desde... hasta 1627. Mss. en la Biblioteca Nacional de Madrid, editados por Carlos Sáez, Alcalá de Henares, Instituto de Estudios Complutenses, 1990. Noticia sobre la arqueta f. ${ }^{0} 1209$ del ms., p. 633. Agradezco a Aurea de la Morena la noticia.

${ }^{15}$ Morán, Miguel y Checa, Fernando, El coleccionismo en España. De la Cámara de las Maravillas a la Galería de pinturas. Madrid, Cátedra. Dio la noticia de la existencia del documento en el Archivo Histórico de Protocolos de Madrid (A.P.M) que se cita en nota 16.

${ }^{16}$ A. P. M., Juan de la Cotera, Protocolo n. ${ }^{\circ} 1811$, año 1599, f. ${ }^{\text {os }} 1495-1697$. El Inventario comienza en f.o $1638 v^{\text {a }}$, después de la relación de su librería. Se describen los bienes existentes en las diversas dependencias incluido el oratorio y a partir del f. 1647 se describen las reliquias. En f. 1656 la nota al margen «hasta leer el inventario de Alcalá por venir».

${ }^{17}$ Furlan, Caterina, «I "Trionfi" della collezione Kress. Una proposta attributiva e divagazione sul tema», en Arte Veneta, XXVII, 1973, 81-90. Marfiles de Graz- 84 y ss., nota 19, figs. 104-109.

${ }_{18}$ Anales Complutenses, cit. en nota 14, Boletín de la Sociedad Española de Excursiones, 1893, p. 17. Tormo y Monzó, Elías, «Alcalá de Henares», Boletín de la Sociedad Española de Excursiones, 1917, pp. 148-149. Rodríguez Marín, Inventario Artístico de la Provincia de Madrid, 1921 (ms. inédito, en depósito en el Departamento de Historia del Arte «Diego Velázquez» del CSIC). Sorribes, »Exposición de Arte Retrospectivo», Boletín de la Sociedad Española de Excursiones, 1926, p. 216. Tormo y Monzó, Elías, Alcalá de Henares, Madrid (1929). Cartillas Exccursionistas, Patronato Nacional de Turismo, Texto similar al de 1917. Estella Marcos, Margarita-Mercedes, La escultura barroca de marfil en España. Las escuelas europeas y las coloniales. Madrid, Departamento de Historia del Arte «Diego Velázquez» del CSIC, 1984, II, cat. n. ${ }^{\circ} 189$, Madrid en el Renacimiento. Alcalá de Henares, octubre-diciembre 1986, p. 307
} 